\title{
Distinct Influences of Urban Villages on Urban Heat Islands: A Case Study in the Pearl River Delta, China
}

\author{
Wei $\mathrm{Wu}^{1,2}$, Hongyan Ren ${ }^{2, *}$, Ming $\mathrm{Yu}^{1, *}$ and Zhen Wang ${ }^{2}$ \\ 1 College of Geographical Science, Fujian Normal University, No.8 Shangsan Road, Fuzhou 350007, China; \\ ww20170820@gmail.com \\ 2 State Key Laboratory of Resources and Environmental Information System, \\ Institute of Geographic Sciences and Natural Resources Research, Chinese Academy of Sciences, \\ 11A Datun Road, Chaoyang District, Beijing 100101, China; wangz.16b@igsnrr.ac.cn \\ * Correspondence: renhy@igsnrr.ac.cn (H.R.); gisyuming@163.com (M.Y.)
}

Received: 26 June 2018; Accepted: 2 August 2018; Published: 6 August 2018

\begin{abstract}
Widely scattered urban villages (UVs) and increasingly serious urban heat islands (UHIs) are common urban problems in highly urbanized regions, especially in the developing countries. However, the influences of UVs on UHIs remain little understood. In this study, different methodologies are performed to retrieve land surface temperature (LST) from thermal bands and the nearest object-oriented method with spectral, texture, shape metrics using ZY-3 high-resolution satellite imagery, and road network data are used to extract UVs and other land-use types in the Guangzhou-Foshan (GF) core areas of Pearl River Delta (PRD). Moreover, the relationship between LST and land-use types is then analyzed on the multiple scales. The results show that five land-use types (vegetation, normal construction land (NCL), UVs, water, and unused land) extracted by the object-oriented method were qualified for subsequent analysis because of satisfactory overall accuracy (0.887) and the Kappa coefficient (0.863). In the GF core areas presenting the most outstanding UHI effect across the PRD region, about $60.5 \%$ of the total area is covered by the impervious surfaces, including NCL (50.4\%) and UVs (10.1\%). The average LST of UVs was $1.89-2.97{ }^{\circ} \mathrm{C}$ lower than that of NCL. According to the average contribution index of thermal effect and the Pearson's correlation coefficients, UVs present a relatively lower contribution to UHI and a weaker warming effect than NCL, but possess a higher contribution to UHI and a stronger warming effect than other land-use types, resulting in some slightly lower LST-valleys in the UVs adjacent to the NCL and distinct LST-peaks of UVs close to vegetation and water on the surface temperature profile lines. This work increases our understanding of the relationship between increasingly serious UHIs and widely distributed UVs, and would be valuable for local authorities to monitor and improve urban environment in metropolitan regions.
\end{abstract}

Keywords: urban village; land-use type; urban heat island; land surface temperature; Pearl River Delta

\section{Introduction}

In recent years, rapid urbanization leads to temperature rise in cities all around the world, forming an urban heat island (UHI). In addition to thermal discomfort, urbanization in the developing world often leads to the problem of informal settlements (e.g., urban villages (UVs) and slums) [1-4]. However, the relationships between these informal urban settlements and abnormal urban thermal environment remain little understood.

Dramatic transformations and reconstruction of urban space were induced by the living and production demands of ever increasing population during the rapid urbanization. A large number of rural villages on edges of cities can become engulfed, leaving the rural settlements intact and scattered 
throughout the urban area [2,5-8]. The development of UVs is neither authorized nor scientifically planned. As a result, UVs are commonly featured by small and dense substandard buildings, absent vegetation coverage, poor sanitary conditions, overcrowded population, and environment pollution $[9,10]$, which attract increasing attention from local urban planners and policymakers who concentrate on the urban renewal and better living environment.

The UHIs are defined by the temperature differences between urban areas and suburban/rural areas [11-15]. Urbanization leads to the transformation of natural landscapes, such as vegetation, water bodies, and agrarian lands, into impervious surfaces, such as construction land. This transformation reduces vegetation evapotranspiration, increases solar radiation absorption, influences the local and regional climate, and gives rise to the UHI [16]. The UHI can be evaluated with air and surface temperatures. The studies of UHI based on air temperatures were mainly to evaluate the intensity of heat islands [17] and simulate the urban temperatures using the Weather Research and Forecasting model $[18,19]$ or Met office Unified model [20]. However, air temperature data are obtained from meteorological stations, and their sparse distribution makes a spatially continuous analysis difficult [21]. Therefore, numerous studies of UHI have been mainly analyzed based on land surface temperature (LST), which can be derived from thermal infrared bands from Landsat [22-24], Moderate Resolution Imaging Spectroradiometer (MODIS) [25] or Advanced Spaceborne Thermal Emission and Reflection Radiometer (ASTER) [26].

The relationship between LST and land use/land cover (LULC) has been a recent research hotspot, which can provide a scientific basis for urban planning and the regulation of regional environment. Numerous studies have shown that the spatiotemporal patterns of UHIs were influenced to various degrees by different land-use types $[22,27,28]$. Among them, the construction land makes the largest contribution to the UHI effect, while forest land, farmland, parks, and water bodies tend to mitigate the UHI effect [29-31]. However, UVs are seldom considered as a separate category from construction land to quantitatively analyze the effect of UVs on UHIs. The main reason is that the mapping of UVs is difficult indeed because unplanned development leads to complex spectral and spatiotemporal patterns [10]. Recently, a few studies of informal settlements in developing countries have used high-resolution remotely sensed data to detect it. Hofmann et al. [8] and Rhinane et al. [32] used an object-oriented approach to detect informal settlements and the precision of the classification was satisfactory. Owen and Wong [33] proposed to detect informal settlements with spectral, texture, geomorphology and road accessibility metrics, and indicated that roads and texture play a key role in depicting the differences between informal and formal settlements.

In this study, we perform to detect UVs based on the object-oriented classification method with spectral, texture, shape metrics using ZY-3 high-resolution remotely sensed imagery and road data. Moreover, we investigate the spatiotemporal patterns of UHIs in the core areas of Guangzhou-Foshan (GF) metropolitan area in the Pearl River Delta (PRD) region, using LST data retrieved from Landsat thermal bands, and explore the contribution of UVs to the UHI effect on the multiple scales. This study would provide support for dynamically monitoring the ecological effect of UVs, and preventing some UVs-related public health risk and the regulation of regional environment.

\section{Study Areas and Data}

The PRD, with the GF metropolitan area as the central region, located in Southern China $\left(21^{\circ} 27^{\prime}-23^{\circ} 56^{\prime} \mathrm{N}, 111^{\circ} 59^{\prime}-115^{\circ} 26^{\prime} \mathrm{E}\right)$, covers a total area of 44,700 square kilometers (Figure 1$)$. It has a marine subtropical monsoon climate, an annual average temperature of $20-22{ }^{\circ} \mathrm{C}$, and an annual rainfall of $1720 \mathrm{~mm}$, with rain and higher temperatures occurring during the same summer period. In 2017, the gross domestic product of the PRD region reached one trillion dollars, and the permanent resident population reached 59.98 million at the end of that year [34]. The PRD, in particular the GF region, has a large number of UVs during the rapid urbanization over the past decades. For example, Guangzhou City has 138 UVs, while Shenzhen has more than 1000, with 200 within the Special Economic Zone [35]. 


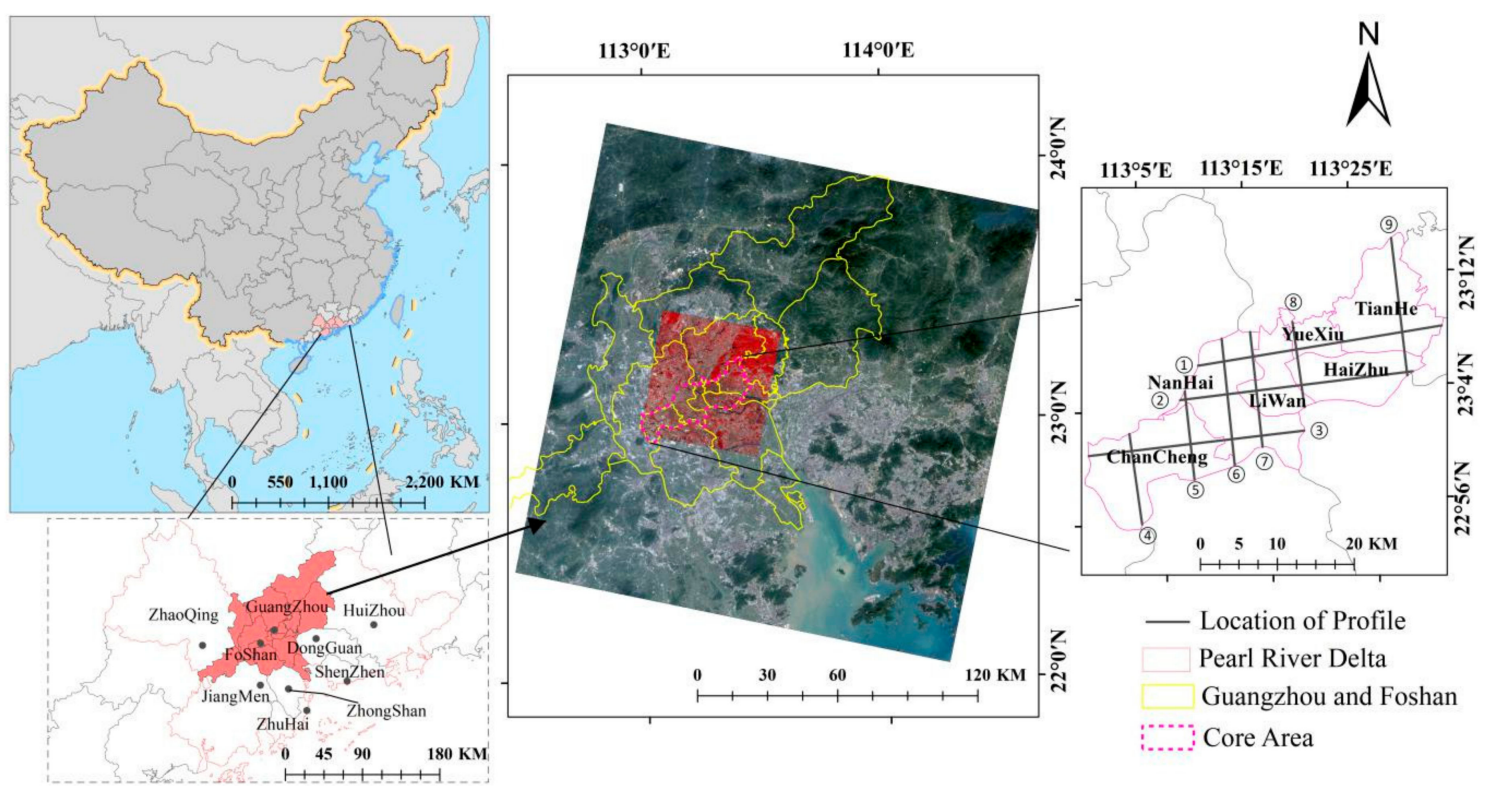

Figure 1. Study areas and satellite data coverage of the Guangzhou-Foshan (GF) in China and locations of profile lines. The data covered consist of the following satellite images (large to small): Landsat 8 Operational Land Imager (OLI) (RGB 321), ZY 3 (RGB 432).

Table 1 outlines the data employed for this study. Before retrieving LST, radiation calibration was performed on the MODIS data, along with geometric correction and cropping of the imagery. For the Landsat $5 / 8$ data (Appendix A), radiometric calibration and Fast Line-of-sight Atmospheric Analysis of Spectral Hypercubes (FLAASH) atmospheric correction were performed. We used ZY-3 satellite data to obtain land-use type data including that of the UVs in the core areas of GF. This data set includes multispectral and panchromatic images with spatial resolutions of $5.8 \mathrm{~m}$ and $2.1 \mathrm{~m}$, respectively. Prior to extraction, we performed geometric corrections and orthorectifications, as well as fusion processing of multispectral and panchromatic images by Pan Sharpening. In addition, road data from Open Street Map (OSM) were used as ancillary data to extract land-use types.

Table 1. Overview of the multitemporal data.

\begin{tabular}{|c|c|c|c|c|}
\hline Data & Date & Resolution & Source & Purpose \\
\hline $\begin{array}{l}\text { MOD021KM } \\
\text { MOD11A1 }\end{array}$ & $\begin{array}{c}22 \text { September } 2011 \\
11 \text { October } 2013 \\
18 \text { October } 2015 \\
23 \text { October } 2017\end{array}$ & $1 \mathrm{~km}$ & $\begin{array}{l}\text { https:/ / ladsweb. } \\
\text { modaps.eosdis.nasa. } \\
\text { gov/search/ }\end{array}$ & $\begin{array}{l}\text { To retrieve LST in the PRD region } \\
\text { and verify the accuracy of LST in } \\
\text { the GF core areas }\end{array}$ \\
\hline Landsat- 5 or 8 & $\begin{array}{l}21 \text { September } 2011 \\
12 \text { October } 2013 \\
18 \text { October } 2015 \\
23 \text { October } 2017\end{array}$ & $30 \mathrm{~m}$ & $\begin{array}{l}\text { http: } \\
\text { //www.gscloud.cn/ }\end{array}$ & $\begin{array}{l}\text { To retrieve more detailed LST in the } \\
\text { GF core areas }\end{array}$ \\
\hline ZY 3 & 14 April 2014 & $5.8 \mathrm{~m} / 2.1 \mathrm{~m}$ & $\begin{array}{l}\text { http://www.cresda. } \\
\text { com/CN/ }\end{array}$ & $\begin{array}{l}\text { To extract UVs and other land-use } \\
\text { types in the GF core areas }\end{array}$ \\
\hline $\begin{array}{l}\text { Road data from } \\
\text { Open Street Map }\end{array}$ & / & / & $\begin{array}{l}\text { http://www. } \\
\text { openstreetmap.org }\end{array}$ & $\begin{array}{l}\text { To serve as auxiliary data for } \\
\text { extracting land use information }\end{array}$ \\
\hline
\end{tabular}

\section{Methods}

As shown in Figure 2, the data-processing procedure involved in this paper mainly includes: (1) retrieve LST based on MODIS data as well as UHI spatial pattern analysis of the PRD region; (2) retrieve LST from Landsat-5 thematic mapper (TM) and Landsat-8 thermal infrared sensor (TIRS), and UHI spatial pattern analysis in typical UHI core areas of PRD; (3) extract UVs and other land-use types in typical UHI core areas; and (4) analyze contribution difference of UVs and other land-use 
types to UHIs. Among them, (1) and (2) relate to retrieval of LST using remote sensing images. We first performed LST retrieval and UHI spatial pattern analysis of the PRD region using remotely sensed imagery with a spatial resolution of $1 \mathrm{~km}$. We also selected a typical UHI core area of PRD (i.e., core areas of GF) and performed LST retrieval and UHI spatial pattern analysis using remote sensing images with a higher spatial resolution $(30 \mathrm{~m})$. Then, we extracted land-use type data from high-resolution remotely sensed imagery for the selected areas (step 3). Finally, we comprehensively analyzed the influence and contribution of each land-use type in the selected areas, especially UVs, to the regional UHI. These procedures will be further described below.

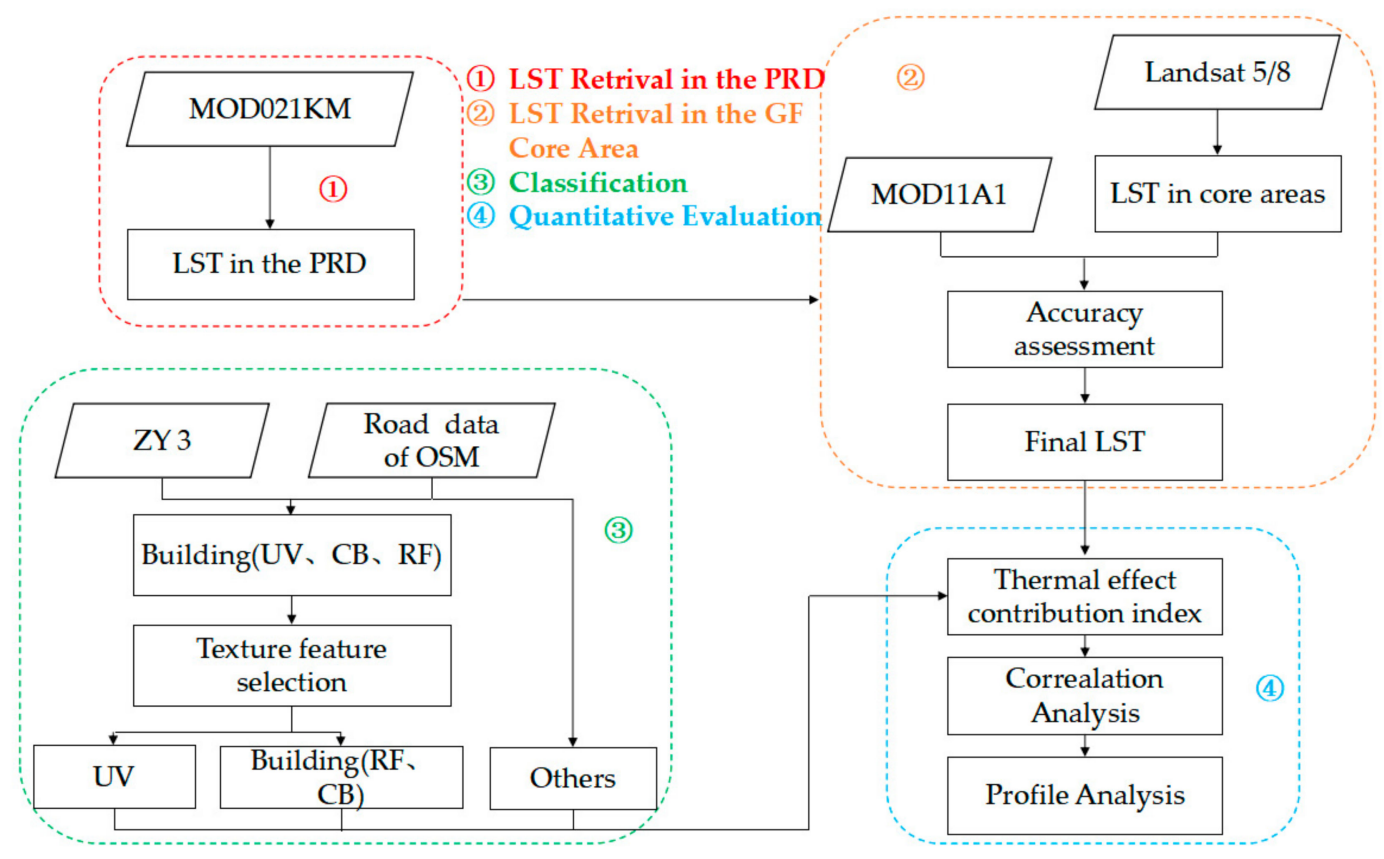

Figure 2. Overall flowchart of exploring the distinctive influences of UVs on urban heat islands (UHIs) (UV: urban village, RF: roofs of factories, $\mathrm{CB}$ : conventional buildings).

\subsection{Land Surface Temperature Retrieval from Thermal Infrared Data}

As mentioned above, this paper discusses two levels of LST retrieval.

(1) LST retrieval in the PRD region. We retrieved LST of the entire PRD region from MODIS thermal infrared bands with a resolution of $1 \mathrm{~km}$ and based on a split window 1 (SW1) algorithm [36]. First, we extracted the 31st, 32nd, 1st, 2nd, and 19th bands of the PRD region. Second, we employed the SW1 method to retrieve LST on 22 September 2011, 11 October 2013, 18 October 2015, and 23 October 2017, respectively. During this process, we adopted estimated brightness temperature, surface emissivity, and atmospheric transmittance. On this basis, due to the errors caused by time phase, we used the standard deviation classification method to eliminate the influence of errors on LST spatial pattern change [37]. The LST was classified into seven categories: lowest, low, sub-low, medium, sub-high, high, and highest temperatures. The zones with the highest, high, and sub-high temperatures were considered as UHI zones. Meanwhile, this study introduced the urban heat island ration index (URI) [38]. The URI was described as shown in Equation (1), where $m$ is the normalization level index; $i$ is the temperature level of urban areas higher than that of the suburb; $n$ is the number of temperature levels of urban areas higher than that of the suburb; $w$ is the weight value, which is the level of the $i$ th level; and $p$ is the percentage of the $i$ th level.

$$
U R I=\frac{1}{100 m} \sum_{i=1}^{n} w_{i} p_{i}
$$


(2) LST retrieval of the typical UHI area. We retrieved LST of typical UHI area from Landsat thermal infrared bands with a resolution of $30 \mathrm{~m}$ (Landsat-5 TM and Landsat-8 TIRS), using different algorithms. First, we used the radiative transfer equation (RT), single channel method (SC) [39], and mono-window algorithm (MW) [40] to retrieve surface temperature from Landsat-5 TM data acquired on 21 September 2011; for the Landsat-8 TIRS data acquired on 18 October 2015, we added a split-window 2 (SW2) algorithm [41]. Because no actually measured data for the surface temperature were used, we adopted the data in the same period of MODIS LST products to verify the retrieval results, in order to select an appropriate retrieval algorithm for Landsat- 8 data acquired on 12 October 2013 and 23 October 2017.

\subsection{Extraction of Land-Use Types}

According to the spatiotemporal pattern of UHI within the PRD region, a typical UHI area is to be selected for extracting land-use type information. The proposed method includes three parts, which are described successively. All analyses were conducted using eCognition Developer 8.7 (Trimble, Trappentreustr, München, Germany).

\subsubsection{Multi-Scale Segmentation of Images Combined with OSM Road Data}

Multi-scale segmentation is a bottom-up method that combines adjacent pixels or small-segmented objects to ensure the minimum average heterogeneity exists between the objects and maximum homogeneity exists between intra-object pixels, in order to achieve image segmentation based on local fusion. The segmentation scale range was set to be 290. Considering the high concentration of buildings in UVs, the shape factor was set to 0.5 , and the smoothness and compactness weights were 0.5. UVs appeared spatially as blocks divided by road networks. In this study, we added adaptive auxiliary data (e.g., OSM road data) in the segmentation process to make it possible to highlight the distinguishing features of the ground objects, while retaining the auxiliary data's adaptability to the spatial characteristics of UVs (Figure 3).

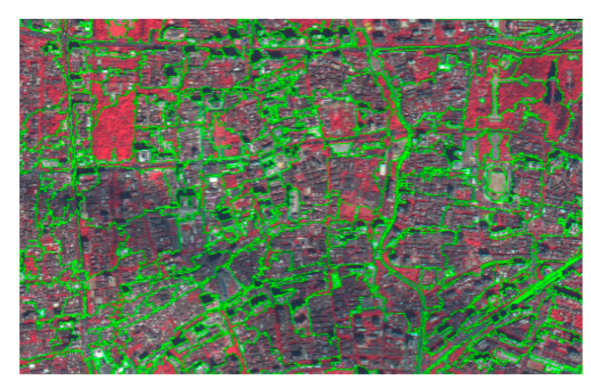

(a)

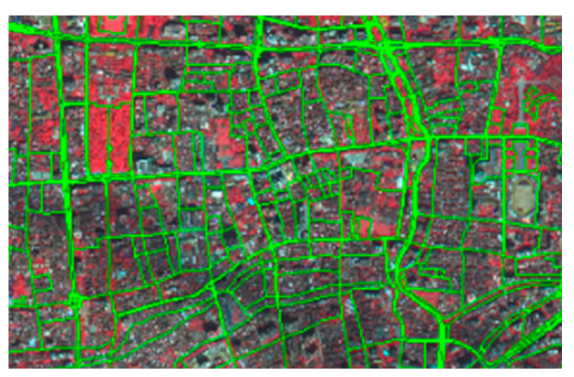

(b)

Figure 3. Comparison of segmentation results. (a) Results without Open Street Map (OSM) road data; (b) results with OSM road data.

\subsubsection{Extraction of Land-Use Types Based on the Nearest Neighbor Method}

Before adopting the nearest neighbor classification method, it is necessary to establish the spectral, spatial, and textural rules of each land-use type.

First, we considered both the spectral and shape features. Among the nine features initially selected, seven were spectral features and inversion indicators, including four multispectral bands (blue, green, red, near-infrared indicators), brightness, maximum difference scaling and normalized difference vegetation index (NDVI); two were object shape features, which were the shape index and length/width. Based on the classification sample and classification system, we optimized these features. Based on the optimal feature dimensions, we calculated the degree of separation of each feature dimension for different object types, and performed a preliminary classification, where we 
divided the land-use types into buildings (e.g., UVs, conventional buildings (CBs), and roofs of factories (RFs)), road, unused land, vegetation, and water.

In addition, we used the gray-level co-occurrence matrix (GLCM) method to extract the texture features of UVs $[42,43]$ to supplement the spectral and shape features to extract UVs from buildings. The GLCM method describes precise textures by analyzing the spatial correlation of gray scales with high computational efficiency. Therefore, based on the preliminary classification of buildings, we collected 30 samples, ten each for UVs, CBs, and RFs (Figure 4).

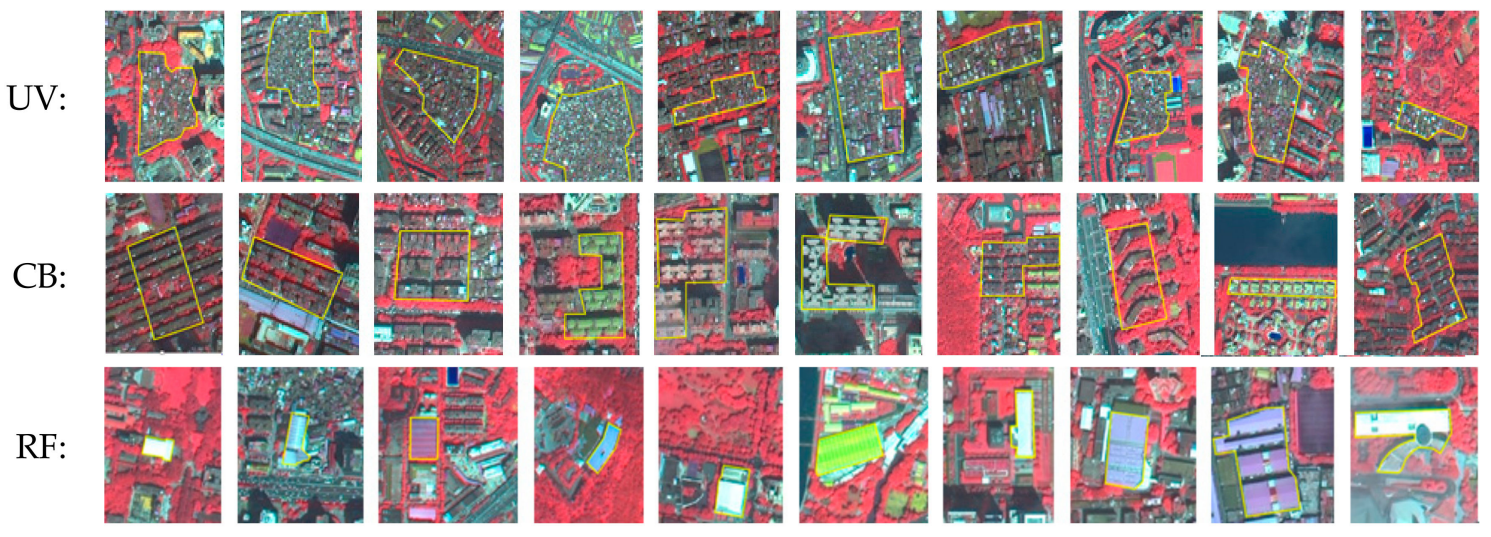

Figure 4. Sample selection: UV, CB and RF (From top to bottom).

To improve the efficiency of UV extraction, we performed $t$-test to compare the differences in eight textures in UVs, CBs, and RFs (Table 2). From the $t$-test results, we can see that the contrast, dissimilarity, StdDev, and mean textures of UV were not significantly different from those of other objects. Therefore, we excluded these four textures. We selected the remaining four textures as alternatives: homogeneity $90^{\circ}$, angular second moment (Ang. 2nd moment) $45^{\circ}$, entropy $90^{\circ}$, and correlation $90^{\circ}$.

Table 2. Student's $t$-test of texture feature differences.

\begin{tabular}{ccccccccc}
\hline Relationship & Homogeneity & Contrast & Dissimilarity & Entropy & $\begin{array}{c}\text { Ang. 2nd } \\
\text { Moment }\end{array}$ & Mean & StdDev & Correlation \\
\hline UV-CB_all & 1.078 & -0.881 & -1.291 & $2.731^{*}$ & -0.538 & 0.686 & -1.514 \\
UV-RF_all & -1.92 & 0.019 & 0.900 & $2.465^{*}$ & -0.611 & 0.384 & -1.251 & -1.822 \\
CB-RF_all & $-2.657^{*}$ & 0.869 & 1.855 & -0.306 & -0.145 & -0.330 & 0.544 & -0.333 \\
UV-CB_0 & 0.859 & -1.463 & -1.911 & $2.685^{*}$ & -2.206 & 0.585 & -1.791 & 0.833 \\
UV-RF_0 & -1.722 & -0.502 & 0.203 & $2.275^{*}$ & -1.795 & 0.602 & -1.273 & 0.251 \\
CB-RF_0 & $-2.765^{*}$ & 0.937 & 1.605 & -0.516 & 0.556 & -0.004 & 0.711 & -0.28 \\
UV-CB_45 & 1.607 & -1.146 & -1.576 & $2.752^{*}$ & $-2.286^{*}$ & 0.739 & -1.527 & -0.159 \\
UV-RF_45 & -1.389 & -0.626 & 0.055 & $2.327^{*}$ & -1.874 & 0.461 & -1.717 & -0.776 \\
CB-RF_45 & -2.197 & 0.663 & 1.258 & -0.562 & 0.744 & -0.256 & 0.432 & -0.327 \\
UV-CB_90 & -0.296 & 1.208 & 0.868 & $2.854^{*}$ & -2.198 & 0.460 & -1.009 & $-3.400 * *$ \\
UV-RF_90 & $-2.330^{*}$ & 1.474 & 2.198 & $2.470 *$ & -2.06 & -0.298 & -0.432 & $-2.489 *$ \\
CB-RF_90 & $-2.405 *$ & 0.037 & 1.864 & -0.544 & 0.754 & -0.913 & 0.384 \\
UV-CB_135 & 2.008 & -0.826 & -1.369 & $2.741^{*}$ & $-2.286 *$ & 0.803 & -1.468 & -1.341 \\
UV-RF_135 & -1.193 & 0.540 & 0.954 & $2.269^{*}$ & -1.801 & 0.469 & -1.452 & -1.74 \\
CB-RF_135 & -1.805 & 1.122 & 2.071 & -0.526 & 0.617 & -0.390 & 0.569 \\
\hline
\end{tabular}

${ }^{*}$ This value is significant at the level of $0.05 .{ }^{* *}$ This value is significant at the level of 0.01 .

Finally, based on the feature space optimization tool of eCognition Developer 8.7, we selected four textures, four spectral bands, and two feature shapes to optimize the feature selection. According to the feature differentiation index, we obtained the optimal feature dimensions and further divided the buildings into UVs, CBs, and RFs. 


\subsubsection{Accuracy Verification}

This paper selected 531 verification sample points by referring to Google Earth imagery and the random point selection methods, and used the Training and Test Area (TTA) Mask file and confusion matrix analysis methods of eCognition Developer 8.7 to evaluate the extraction accuracy of land-use type data. The kappa coefficient and producer's accuracy/user's accuracy were computed for the assessment. The producer's/user's accuracy is the ratio of the number of correctly classified samples to the number of reference/classification samples. The kappa coefficient was written as shown in Equation (2):

$$
\text { Kappa }=\frac{N \sum_{i=1}^{r} x_{i i}-\sum_{i=1}^{r}\left(x_{i+} \times x_{+i}\right)}{N^{2}-\sum_{i=1}^{r}\left(x_{i+} \times x_{+i}\right)}
$$

where $r$ is the number of rows in the confusion matrix of classification; $x_{i i}$ is the number of along the diagonal; $x_{i+}$ is the total number of row $i ; x_{+i}$ is the total number of column $i$; and $N$ is the total number of cells.

\subsection{Evaluation of Contributions of Different Land-Use Types to Heat Island Effects}

This study aims to use the contribution index of thermal effect to quantitatively evaluate the contribution of different land-use types to the heat island effect in the core urban area of GF region. The contribution index of thermal effect $\left(H_{i}\right)$ refers to the degree of impact that the surface temperatures of different land-use types have on the region's average temperature, i.e., the contribution of each land-use type to the region's heat island effect. $H_{i}$ and the contribution of the initial heat island effect $\left(H_{i}^{\prime}\right)$ were shown in Equations (3) and (4):

$$
\begin{gathered}
H_{i}=\frac{H_{i}^{\prime}}{\sum_{i=1}^{n} H_{i}^{\prime}} * 100 \% \\
H_{i}^{\prime}=\frac{\sum_{j=1}^{n}\left(T_{i j}-T_{a 0}\right) * n_{i}}{T_{a 0} N}
\end{gathered}
$$

where $T_{i j}$ is the temperature of the $j$ th pixel with temperatures above the average regional temperature in the land category $i ; T_{a 0}$ represents the average temperature of the region during imaging; $n_{i}$ is the number of pixels in the land category $i$, of which the temperature is higher than the average regional temperature; and $N$ represents the region's land area. To simplify the comparison, we performed data normalization and obtained the contribution indices of different land-use types.

In addition, in order to further study the relationships of surface temperature to UVs, normal construction land, water, and vegetation, this paper randomly selected 40 points inside the UVs and generated buffers with radii of $0.2,0.5,0.8$, and $1 \mathrm{~km}$. We analyzed the relationship between the area proportion of different land-use types and surface temperature in each buffer zone, using Pearson's correlation coefficients.

\subsection{Profile Analysis of Land Surface Temperature in Typical Urban Heat Island Areas}

The temperature profile analysis can be used to explore the internal structure of the heat islands in downtown and urbanized areas. However, it can also be used for analyzing the differences in LST between urban and rural lands, as well as the effect of urban expansion on heat islands [44]. Using ArcGIS 10.3 (ESRI, Redlands, CA, USA) software, this study randomly selected nine surface temperature profile lines from west to east and from north to south (locations of profile lines and serial numbers of profile lines in Figure 1). Based on the ZY-3 high-resolution remotely sensed imagery, we subdivided the land-use types on each profile line and marked them with different colors, so that we could more intuitively and accurately analyze the differences in surface temperature profile lines between different land-use types. 


\section{Results}

\subsection{Spatiotemporal Patterns of Urban Heat Island}

The LST exhibited clear spatial differentiation characteristics in the PRD region. As illustrated in Figure 5, the zones with the highest, high, and sub-high temperatures (i.e., UHI zones) were mainly distributed in four areas, the GF core areas, the Guangzhou-Dongguan city interface, Shenzhen, and the Zhongshan-Zhuhai city interface. Meanwhile, the lowest and low temperature zones were mainly distributed in Zhaoqing, Northern Huizhou, and Northern Guangzhou. The spatial pattern of LST in the PRD region has not changed significantly during 2011-2017. Accordingly, the core areas of GF were considered as a typical area for further analysis of UHI in detail.

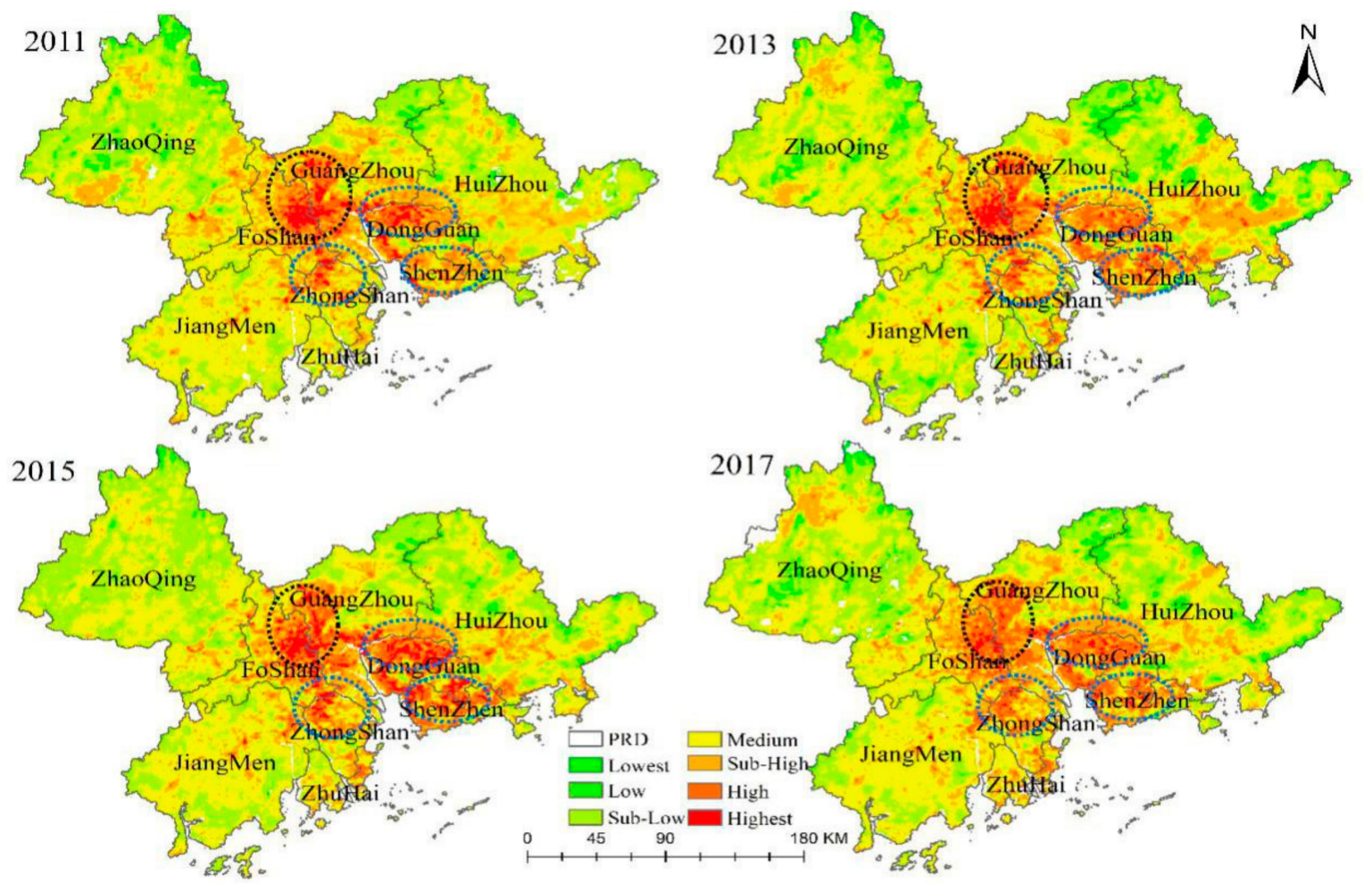

Figure 5. Spatiotemporal patterns of land surface temperature (LST) in the Pearl River Delta (PRD) region with four typical areas on 22 September 2011, 11 October 2013, 18 October 2015 and 23 October 2017.

The comparisons of the LST retrieval results using different algorithms and the MODIS LST products were shown in the Supplementary Files (the scatter plots in Figures S1 and S2 and statistic parameters in Table S1). Similarly, UHI zones in the GF core areas were also spatially differentiated. As shown in Figure 6, these zones were observed in each administrative district, like Liwan, Nanhai and Changchneg Districts. In particular, UHI zones in 2015 and 2017 were spatially clustered near the interface of Liwan and Nanhai Districts. In contrast, the zones with lower temperature, rather than those with the sub-low temperature level, were mainly distributed in the northern part of Tianhe District, the eastern part of Haizhu District, and the western part of Chancheng District. Meanwhile, the UHI effect presented an overall downward trend according to the URI decreasing to 0.2031, as well as the slightly decline of the UHI area proportion from 32.06 to $30.34 \%$ (Table 3). These results showed that the UHI effect in the GF core areas was mitigated to some degree in the past 7 years. 


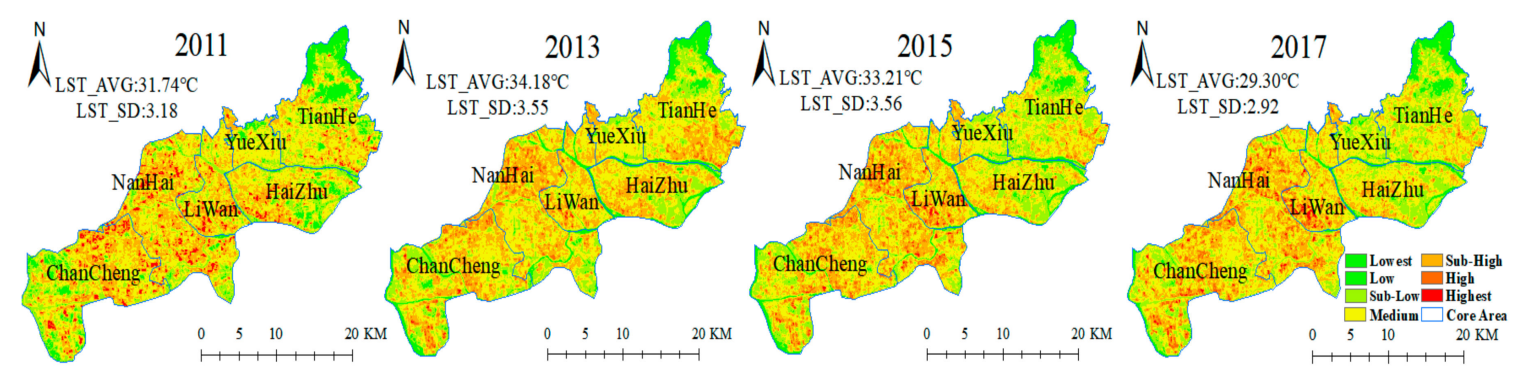

Figure 6. Spatiotemporal patterns of LST in the core urban areas of GF on 22 September 2011, 11 October 2013, 18 October 2015 and 23 October 2017. (LST_AVG: average LST; LST_SD: LST standard deviation).

Table 3. Variation of graded area of LST in the core areas of GF.

\begin{tabular}{|c|c|c|c|c|c|c|c|c|}
\hline \multirow{2}{*}{ Class } & \multicolumn{2}{|c|}{2011} & \multicolumn{2}{|c|}{2013} & \multicolumn{2}{|c|}{2015} & \multicolumn{2}{|c|}{2017} \\
\hline & Area $/ \mathrm{km}^{2}$ & Per/\% & Area $/ \mathrm{km}^{2}$ & Per/\% & Area $/ \mathrm{km}^{2}$ & Per/\% & Area $/ \mathrm{km}^{2}$ & Per $/ \%$ \\
\hline Lowest & 6.525 & 1.05 & 5.36 & 0.86 & 1.26 & 0.20 & 2.60 & 0.42 \\
\hline Low & 34.21 & 5.48 & 45.77 & 7.33 & 46.57 & 7.46 & 38.01 & 6.09 \\
\hline Sub-low & 127.51 & 20.43 & 106.77 & 17.11 & 121.64 & 19.49 & 129.97 & 20.83 \\
\hline Medium & 255.79 & 40.99 & 261.12 & 41.84 & 255.47 & 40.94 & 264.12 & 42.32 \\
\hline Sub-high & 176.71 & 28.32 & 176.07 & 28.21 & 162.47 & 26.04 & 149.22 & 23.91 \\
\hline High & 22.03 & 3.53 & 27.66 & 4.43 & 33.68 & 5.40 & 35.48 & 5.68 \\
\hline Highest & 1.30 & 0.21 & 1.31 & 0.21 & 2.95 & 0.47 & 4.70 & 0.75 \\
\hline URI & \multicolumn{2}{|c|}{0.2346} & \multicolumn{2}{|c|}{0.2416} & \multicolumn{2}{|c|}{0.2370} & \multicolumn{2}{|c|}{0.2031} \\
\hline
\end{tabular}

\subsection{Results and Analysis of Land-Use Types}

According to the overall accuracy (0.876) and the Kappa coefficient (0.851) (Table 4), the land-use types were well extracted from the ZY-3 high-resolution remote sensing images. As far as the producer's and user's accuracy were concerned, the UVs possessed slightly lower accuracies $(77.6 \%$ and $84.6 \%)$ than those of vegetation $(94.7 \%$ and $98.2 \%)$, water $(98.6 \%$ and $97.2 \%)$, and roads $(89.0 \%$ and $87.2 \%)$, although the omission and commission of UVs have been appropriately controlled by the texture selection procedure. In a word, the present extraction accuracy can meet the demands of subsequent analysis. Then, the roads, CBs, and RFs were combined into the normal construction land (NCL) type, resulting in the five major land-use types including NCL, UVs, unused land, vegetation, and water.

Table 4. Confusion matrix of land classification.

\begin{tabular}{ccccccccc}
\hline Class & Vegetation & Road & Water & Unused Land & UV & CB & RF & Sum \\
\hline Vegetation & 54 & 0 & 0 & 0 & 1 & 0 & 0 & 55 \\
Road & 1 & 89 & 0 & 0 & 5 & 4 & 3 & 102 \\
Water & 0 & 0 & 143 & 2 & 2 & 0 & 0 & 147 \\
Unused land & 1 & 4 & 2 & 42 & 4 & 8 & 1 & 62 \\
UV & 0 & 5 & 0 & 2 & 66 & 5 & 0 & 78 \\
CB & 1 & 2 & 0 & 1 & 2 & 23 & 0 & 28 \\
RF & 0 & 0 & 0 & 1 & 5 & 4 & 48 & 58 \\
Sum & 57 & 100 & 145 & 48 & 85 & 44 & 52 & 531 \\
Producer's accuracy & 0.947 & 0.890 & 0.986 & 0.875 & 0.776 & 0.523 & 0.923 & \\
User's accuracy & 0.982 & 0.872 & 0.972 & 0.677 & 0.846 & 0.821 & 0.827 & \\
\hline Overall accuracy & & & & \\
Kappa & & & & 0.876 & & & & \\
\hline
\end{tabular}


In terms of urban space, the GF core areas were highly urbanized. According to the area ratio of five land-use types as illustrated in Figure 7, more than $60 \%$ of the core areas were covered by impervious surfaces including NCL (50.1\%) and UVs (10.4\%). In comparison, about $31 \%$ of this region was covered by permeable surfaces (vegetation, $28.0 \%$; water area, $8.4 \%$ ). Similar to the spatial patterns of UHI zones, UVs also appeared in each administrative district. There were several zones with spatially clustered UVs as labeled by black dotted ovals in Figure 7, including the zones near the Pearl River fork between Yuexiu and Haizhu Districts, the Wangjiegang forest park in Chancheng District, the Pingzhou Branch of Provincial People's Hospital in Nanhai District. These results indicated that the GF core areas were featured by widely distributed UVs.

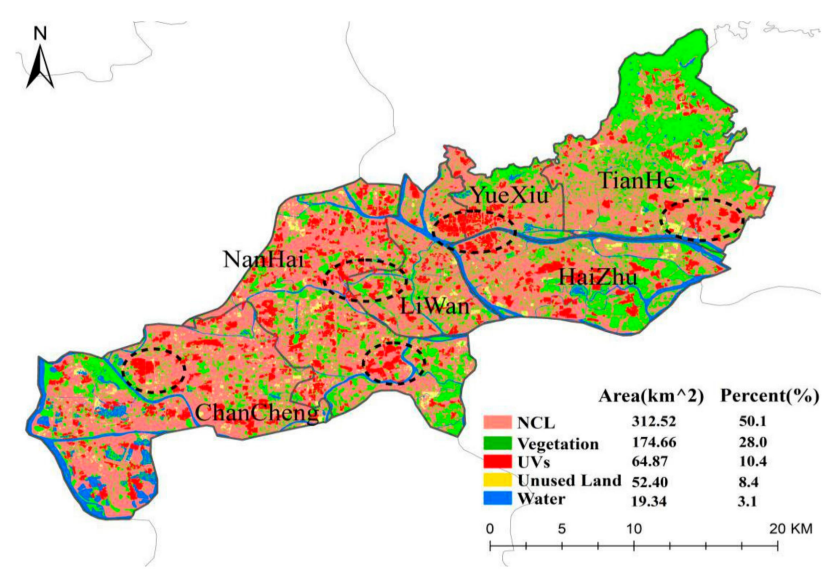

Figure 7. Classification result in the core urban areas of GF.

\subsection{Contributions of Different Land-Use Types to Urban Heat Island}

The average surface temperature and UHI contribution index $\left(H_{i}\right)$ varied with the land-use types across the GF core areas. The five land-use types were ranked from the highest to the lowest surface temperature values as follows (Table 5): NCL > unused land $>$ UVs $>$ vegetation $>$ water, which persisted during 2011-2017. The average surface temperature of NCL was $1.89-2.97^{\circ} \mathrm{C}$ higher than that of UVs. In comparison, the sequence of $H_{i}$ for these land-use types was slightly different: $\mathrm{NCL}>\mathrm{UVs}>$ vegetation $>$ unused land $>$ water, and this order also remained unchanged in this period. These results showed that different land-use types made different contributions to the spatial patterns of UHI across the GF core areas, and NCL contributed the most to the UHI effect across the GF core urban areas, followed by UVs.

Table 5. Average surface temperatures and thermal effect contribution indices of thermal effect for different land-use types (AVG: average; SD: standard deviation).

\begin{tabular}{ccccccccc}
\hline \multirow{2}{*}{ Class } & \multicolumn{2}{c}{$\mathbf{2 0 1 1}$} & \multicolumn{2}{c}{$\mathbf{2 0 1 3}$} & $\mathbf{2 0 1 5}$ & \multicolumn{2}{c}{$\mathbf{2 0 1 7}$} \\
\cline { 2 - 8 } & AVG $\pm \mathbf{S D}$ & $\boldsymbol{H}_{\boldsymbol{i}} / \%$ & AVG $\pm \mathbf{S D}$ & $\boldsymbol{H}_{\boldsymbol{i}} / \%$ & AVG $\pm \mathbf{S D}$ & $\boldsymbol{H}_{\boldsymbol{i}} / \%$ & AVG $\pm \mathbf{S D}$ & $\boldsymbol{H}_{\boldsymbol{i}} / \%$ \\
\hline Vegetation & $29.42 \pm 2.61$ & 3.00 & $32.57 \pm 2.93$ & 2.90 & $31.21 \pm 2.76$ & 1.91 & $27.78 \pm 2.41$ & 1.30 \\
Water & $28.33 \pm 1.82$ & 0.01 & $28.82 \pm 2.84$ & 0.01 & $28.06 \pm 2.86$ & 0.01 & $25.97 \pm 1.84$ & 0.01 \\
NCL & $34.88 \pm 2.66$ & 92.85 & $38.71 \pm 2.60$ & 92.67 & $36.80 \pm 2.91$ & 93.57 & $32.59 \pm 2.47$ & 95.03 \\
UVs & $32.99 \pm 1.72$ & 3.78 & $35.74 \pm 1.74$ & 4.08 & $34.74 \pm 1.81$ & 4.17 & $30.31 \pm 1.50$ & 3.29 \\
Unused Land & $33.08 \pm 2.71$ & 0.36 & $35.56 \pm 2.60$ & 0.34 & $35.75 \pm 2.37$ & 0.34 & $30.40 \pm 2.02$ & 0.37 \\
\hline
\end{tabular}

In addition, the average regional LST was correlated with the proportions of area of various land-use types. According to the Pearson's correlation coefficients in Table 6, the average LST values decreased significantly when the proportion of area covered by the permeable surfaces (vegetation and water) increased in the UV-centered buffer zones. Meanwhile, surface temperature increased 
remarkably when those of area covered by the impervious surfaces (NCL and UVs) were higher in these buffer zones. In contrast to the significantly positive influence of NCL on the surface temperature, the contribution of UVs was not remarkable, which means that UVs and NCL possessed weak and strong warming effects in the core areas, respectively.

Lastly, the influences of land-use types on the spatial patterns of UHI could be further understood according to the analysis of the profile lines across the GF core areas.

Table 6. Pearson's correlation coefficients of surface temperature and area ration of different land-use types.

\begin{tabular}{ccccc}
\hline Year & Impervious Surfaces & NCL & UVs & Permeable Surfaces \\
\hline 2011 & $0.604^{* *}$ & $0.292^{* *}$ & 0.194 & $-0.680^{* *}$ \\
2013 & $0.943^{* *}$ & $0.220^{* *}$ & $0.189^{*}$ & $-0.694^{* *}$ \\
2015 & $0.525^{* *}$ & $0.182^{*}$ & 0.147 & $-0.591^{* *}$ \\
2017 & $0.427^{* *}$ & $0.230^{* *}$ & 0.060 & $-0.500^{* *}$ \\
\hline
\end{tabular}

* This value is significant at the level of 0.05 . ${ }^{* *}$ This value is significant at the level of 0.01 . NCL and UVs were classified into the category of impervious surfaces while the permeable surfaces included water and vegetation.

On the LST profile line 1 (see the other profile lines in the Supplementary Files in Figure S3), the surface temperature values for all 5 land-use types formed a waving curve from 2011 to 2017 (Figure 8). The average temperature of all profile lines in 2011-2017 were ranked from the highest to the lowest as follows: NCL > UVs > Vegetation > Water. As the largest contributor to the UHI effect, NCL was mainly indicated by outstanding peaks on the profile lines. Water and vegetation appeared as obvious valley-shapes due to their relatively low surface temperature. UVs tended to be featured by clear valley shapes when they were adjacent to NCL (labeled by blue dotted rectangles) on the profile lines. However, when adjacent to the vegetation and water bodies (labeled by purple dotted rectangles), UVs showed a small peak shape on the profile lines, indicating an UHI phenomenon. In the GF core areas, the areas with small peak shapes were, for example, near the Zhujiang fork between Yuexiu and Haizhu Districts, nearby the Guangjun Business Center in Haizhu District, and near Huolushan Park in Tianhe Park. The results indicated that UVs were widespread and mainly surrounded by NCL, so that the slight but clear surface temperature valleys could be presented in this highly urbanized region.

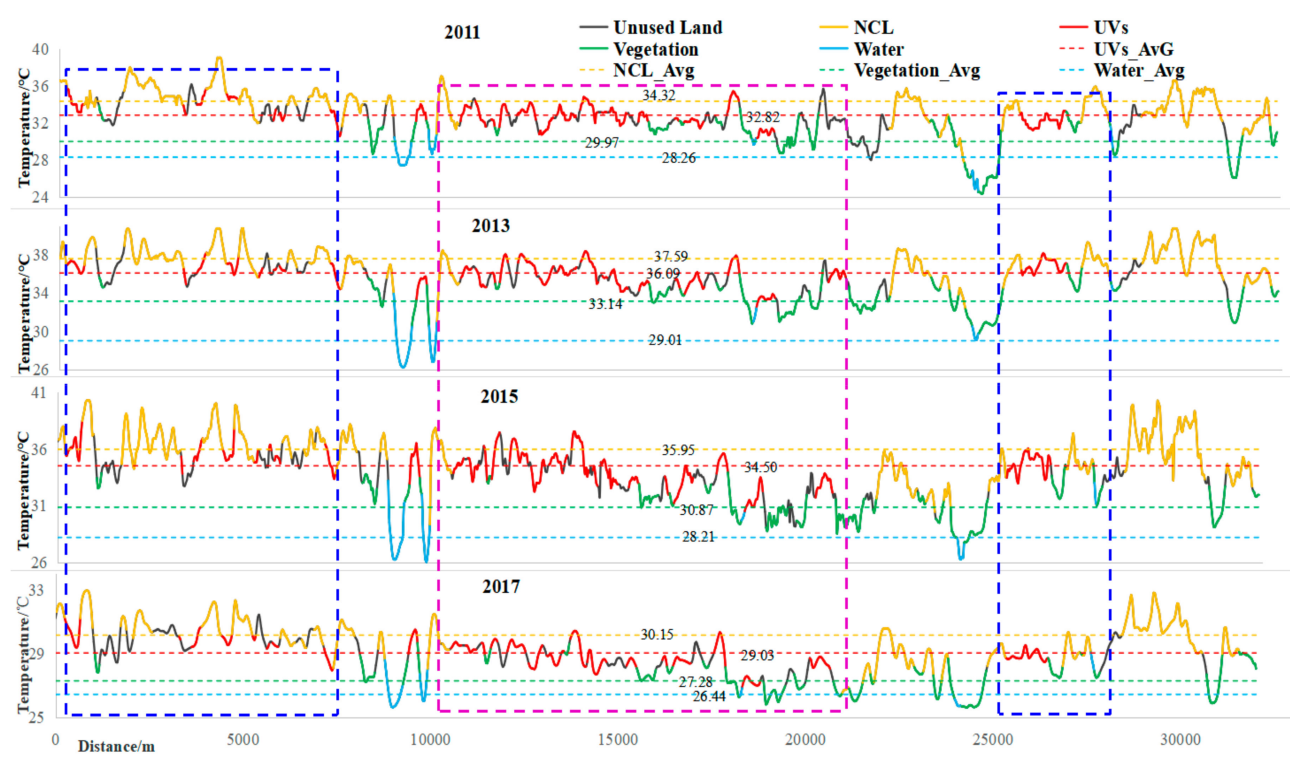

Figure 8. Profile analysis results of Line 1 (NCL_AVG: average surface temperature of normal construction land; UVs_AVG: average surface temperature of UVs; Vegetation_AVG: average surface temperature of vegetation; Water AVG: average surface temperature of water). 


\section{Discussion}

To our knowledge, our study concentrated on the effects of widely scattered UVs on increasingly serious UHIs in some metropolitan regions for the first time. In this study, we analyzed the influences of UVs, as well as other land-use types, on the spatiotemporal patterns of UHI in the GF core areas by means of multi-source remote sensing data. This study will help to understand the spatiotemporal patterns of UHI in the urban area with numerous UVs, which can provide local environmental and hygienic authorities with useful clues about environmental enhancement and related public health management.

It has been proved that the object-oriented classification method is an effective solution for detecting the informal settlements (e.g., UVs and slums) [8,32], as well as the scenarios-based classification [10], landscape metrics and transfer learning [9]. However, the development of UVs in China has seldom been reported or analyzed using the object-oriented classification method. In addition, image segmentation is still difficult and challenging [45-47]. Numerous studies indicated that image segmentation efficiency was improved when adding some auxiliary data, such as digital elevation models, cadastral, population, or road network [48,49]. In our study, OSM road data was employed as auxiliary data for improving the efficiency of ZY-3 high-resolution remotely sensed imagery segmentation. Moreover, the extraction of texture using the GLCM method was used to enrich the features of ground objects to overcome the disadvantages of shape and spectral features in identifying UVs from CBs and RFs. As a result, UVs and other land-use types were effectively extracted in the GF core areas with an overall accuracy of 0.876 and the Kappa coefficient of 0.851 . The results indicated that the nearest object-oriented method had strong adaptability and high efficiency and could be considered as an alternative approach to detect UVs in China.

The UHI is an outstanding environment problem due to its profound influence on the lives of urban residents, and some research have focused on how to mitigate it [50-53]. In our study, the UHI effect in the GF core areas was mitigated to some degree in the past 7 years. It is due to the fact that the joint GF development that has taken place in recent years has been accompanied by continuous implementation of policies protecting and restoring green spaces and water areas for the improvement of the ecological structure of the GF core areas. In addition, previous studies have pointed out that the construction land is a predominant contributor and an important influencing factor of UHIs, while vegetation and water can mitigate heat islands in the urban area $[22,27,28]$. These findings are similar to our study that NCL presented the highest ground temperature, as well as the largest contribution to UHIs in the GF core areas. In comparison, UVs, as a specific land-use type separated from the construction land, possessed $1.89-2.97^{\circ} \mathrm{C}$ lower surface temperature and a relatively weaker warming effect, which may be related to the buildings' tile roofs of UVs with higher reflectance than cement roofs or their unique roofs with many potted plants [54]. In addition, the slight but clear surface temperature valleys could be presented from surface temperature profile analysis results. These results implied that the specific urban settlement (i.e., UVs) should be considered as a separate land-use type when the influences of LULC on UHIs were to be investigated. It would supply useful reference for monitoring and managing urban environment.

In fact, UVs are widely distributed in not only the GF core areas but also the other cities (e.g., Shenzhen City) in the highly urbanized PRD region. In addition to the relatively lower temperature, UVs are commonly featured by poor sanitation, overcrowding population, low coverage public health services $[9,10]$, resulting in a high environmental suitability for some biological vectors (e.g., Aedes Albopictus), as well as the vector-borne diseases in these urban regions [55,56]. Accordingly, UVs should be heavily emphasized due to their serious environmental health risk. Together with the quick detection of UVs by using remote sensing, the unique influences of UVs on UHIs investigated in this study would provide meaningful clues for monitoring biological vectors, as well as preventing and controlling UVs-related infection in these urban areas with numerous UVs.

A few limitations of this study warrant mention. First, much earlier UVs information before 2012 should be obtained for the future analysis of the spatiotemporal variations of UVs, although the 
urban reconstruction focusing on the demolishing UVs was slowed down after the 2010 Asian Games. Additionally, the extraction accuracy of all the land-use types could be further improved through adding many more auxiliary data (i.e., openly accessible point of interest (POI) data). Finally, ground temperature at night may be spatially different from that of daytime across the urban area and the analysis of their difference could be conducted in future research to provide more meaningful support for monitoring and regulating urban environment.

\section{Conclusions}

In summary, this paper draws the following conclusions:

(1) This study, for the first time, systematically verified the distinctive effect of the spatial distribution of UVs on UHI, which was mainly characterized by the lower surface temperature and relatively weaker warming effect of UVs than those of NCL.

(2) Multi-scale segmentation combined with road data and the extraction of UVs based on the nearest neighbor method with spectral, shape and texture metrics provided methodological reference for the detection of informal settlement (e.g., slums or UVs) in future research.

(3) This study provides theoretical support for the dynamic monitoring, transformation, and redevelopment of UVs, preventing some UVs-related public health risk as well as the regulation of the regional environment.

Supplementary Materials: The following are available online at http:/ /www.mdpi.com/1660-4601/15/8/1666/ s1. Figure S1: Scatter plot of different methodologies to retrieve surface temperature from Landsat-5 TM and MODIS LST product on 21 September 2011, Figure S2: Scatter plot of different methodologies to retrieve surface temperature from Landsat- 8 TIRS and MODIS LST product on 18 October 2015, Figure S3: Temperature profile analysis results of Line 2-9, Table S1: Different methodologies to retrieve surface temperature from Landsat-5 TM and Landsat-8 TIRS.

Author Contributions: H.R. conceived and designed the research. W.W. analyzed and performed the experiments, and wrote the first draft of the article. H.R. and M.Y. reviewed and edited the first draft. Z.W. contributed to detecting UVs.

Funding: This research was funded by the National Natural Science Foundation of China (Grant No. 41571158), the National key research and development program (No. 2016YFC1201305-03) and the state key laboratory of resources and environment information systems, independent innovation project (No. O8R8B6A0YA).

Acknowledgments: The authors are grateful to the anonymous reviewers and editors for their valuable comments and suggestions on improving the quality of the paper and are also grateful to the ZY-3, MODIS and Landsat 5/8 science teams for their excellent and accessible data products. We would like to thank all the project partners and members for their support.

Conflicts of Interest: The authors declare no conflicts of interest.

\section{Appendix A}

List of Landsat-5/8 images used for LST retrieval according to their path, row, date (dd/mm/yyyy) and scene name.

Table A1. List of Landsat images used in this study.

\begin{tabular}{cccc}
\hline Path & Row & Date & Landsat Scene \\
\hline 122 & 44 & $21 / 09 / 2011$ & LT51220442011264BKT00 \\
122 & 44 & $12 / 10 / 2013$ & LC81220442013285LGN01 \\
122 & 44 & $18 / 10 / 2015$ & LC81220442015291LGN00 \\
122 & 44 & $23 / 10 / 2017$ & LC81220442017296LGN00 \\
\hline
\end{tabular}


Table A2. List of acronyms.

\begin{tabular}{cccc}
\hline Acronyms & Acronyms of Full Name & Acronyms & Acronyms of Full Name \\
\hline ASTER & $\begin{array}{c}\text { Advanced Spaceborne Thermal Emission and } \\
\text { Reflection Radiometer }\end{array}$ & OSM & Open Street Map \\
\hline AVG & Average & PRD & Peral River Delta \\
\hline CB & Conventional buildings & RF & Roofs of factories \\
\hline FLAASH & Fast Line-of-sight Atmospheric Analysis of & RT & Radiative transfer equation \\
\hline GF & Guangzhou and Foshan & SC & Single channel method \\
\hline GLCM & Gray level co-occurrence matrix & SD & Standard deviation \\
\hline LST & Land surface temperature & SW & Split window \\
\hline LULC & Land use/Land cover & TIRS & Thermal Infrared Sensor \\
\hline MW & Mono window & TM & Thematic mapper \\
\hline MODIS & Moderate resolution Imaging Spectroradiometer & TTA & Training and Test Area \\
\hline NCL & Normal construction land & UHI & Urban heat island \\
\hline NDVI & Normalized difference vegetation index & URI & Urban ratio index \\
\hline OLI & Operational Land Imager & UVs & Urban villages \\
\hline
\end{tabular}

\section{References}

1. Lopez, M.E. Slums of the World: The Face of Urban Poverty in the New Millennium? UN-Habitat: Nairobi, Kenya, 2003.

2. Chung, H. Building an image of villages-in-the-city: A clarification of China's distinct urban spaces. Int. J. Urban Reg. Res. 2010, 34, 421-437. [CrossRef] [PubMed]

3. Li, Z.; Wu, F. Residential satisfaction in China's informal settlements: A case study of Beijing, Shanghai, and Guangzhou. Urban Geogr. 2013, 34, 923-949. [CrossRef]

4. Ghasempour, A. Informal settlement: Concept, challenges, and intervention approaches. Spec. J. Arch. Constr. 2015, 1, 10-16.

5. Hao, P.; Hooimeijer, P.; Sliuzas, R.; Geertman, S. What drives the spatial development of urban villages in China? Urban Stud. 2013, 50, 3394-3411. [CrossRef]

6. Taubenböck, H.; Kraff, N.J. The physical face of slums: A structural comparison of slums in Mumbai, India, based on remotely sensed data. J. Hous. Built Environ. 2014, 29, 15-38. [CrossRef]

7. Goebel, A. Sustainable urban development? Low-cost housing challenges in South Africa. Habitat Int. 2007, 31, 291-302.

8. Hofmann, P.; Strobl, J.; Blaschke, T.; Kux, H. Detecting informal settlements from QuickBird data in Rio de Janeiro using an object-based approach. In Object-Based Image Analysis; Lecture Notes in Geoinformation and Cartography; Blaschke, T., Land, S., Hay, G., Eds.; Springer: Berlin/Heidelberg, Germany, 2008; pp. 531-553.

9. Liu, H.; Huang, X.; Wen, D.; Li, J. The use of landscape metrics and transfer learning to explore urban villages in China. Remote Sens. 2017, 9, 365. [CrossRef]

10. Huang, X.; Liu, H.; Zhang, L. Spatiotemporal detection and analysis of urban villages in mega City regions of China using high-resolution remotely sensed imagery. IEEE Trans. Geosci. Remote Sens. 2015, 53, 3639-3657. [CrossRef]

11. Oke, T.R. Inadvertent climate modification. In Boundary Layer Climates, 2nd ed.; Methuen and Co.: New York, NY, USA, 1987; pp. 262-303.

12. Bryson, R.A.; Ross, J.E. The Climate of the City: Urbanization and Environment; Duxbury Press: Belmont, CA, USA, 1972.

13. Chandler, T.J. The Climate of London; Hutchison of London: London, UK, 1965; p. 287.

14. Landsberg, H.E. The Urban Climate, 1st ed.; Academic Press: New York, NY, USA, 1981.

15. Lowry, W.P. Empirical estimation of urban effects on climate: A problem analysis. J. Appl. Meteorol. 1977, 16, 129-135. [CrossRef]

16. Yao, R.; Wang, L.; Huang, X.; Niu, Z.; Liu, F.; Wang, Q. Temporal trends of surface urban heat islands and associated determinants in major Chinese cities. Sci. Total Environ. 2017, 609, 742-754. [CrossRef] [PubMed]

17. Amorim, M.; Dubreuil, V. Intensity of Urban Heat Islands in Tropical and Temperate Climates. Climate 2017, 5, 91. [CrossRef] 
18. Miao, S.; Chen, F.; Lemone, M.A.; Tewari, M. An Observational and Modeling Study of Characteristics of Urban Heat Island and Boundary Layer Structures in Beijing. J. Appl. Meteorol. Climatol. 2009, 48, 484-501. [CrossRef]

19. Sharma, A.; Fernando, H.J.S.; Hamlet, A.F.; Hellmann, J.J.; Barlage, M.; Chen, F. Urban meteorological modeling using WRF: A sensitivity study. Int. J. Climatol. 2017, 37, 1885-1900. [CrossRef]

20. Bohnenstengel, S.I.; Evans, S.; Clark, P.A.; Belcher, S.E. Simulations of the London urban heat island. Q. J. R. Meteorol. Soc. 2011, 137, 1625-1640. [CrossRef]

21. Shen, H.; Huang, L.; Zhang, L.; Wu, P.; Zeng, C. Long-term and fine-scale satellite monitoring of the urban heat island effect by the fusion of multi-temporal and multi-sensor remote sensed data: A 26-year case study of the city of Wuhan in China. Remote Sens. Environ. 2016, 172, 109-125. [CrossRef]

22. Qian, L.; Ding, S. Influence of land cover change on land surface temperature in Zhujiang Delta. Acta Geogr. Sin. 2005, 5, 761-770.

23. Zhao, M.; Cai, H.; Qiao, Z.; Xu, X. Influence of urban expansion on the urban heat island effect in Shanghai. Int. J. Geogr. Inf. Syst. 2016, 30, 2421-2441. [CrossRef]

24. Zhang, H.; Qi, Z.F.; Ye, X.Y.; Cai, Y.; Ma, W.; Chen, M. Analysis of land use/land cover change, population shift, and their effects on spatiotemporal patterns of urban heat islands in metropolitan Shanghai, China. Appl. Geogr. 2013, 44, 121-133. [CrossRef]

25. Rao, S.; Zhang, H.Y.; Jin, T.T.; Dou, H.Y. The spatial character of regional heat island in Pearl River Delta using MODIS remote sensing data. Geogr. Res. 2010, 29, 127-136.

26. Duan, S.B.; Li, Z.L.; Cheng, J.; Leng, P. Cross-satellite comparison of operational land surface temperature products derived from MODIS and ASTER data over bare soil surfaces. ISPRS J. Photogramm. 2017, 126, 1-10. [CrossRef]

27. Wu, H.; Ye, L.P.; Shi, W.Z.; Clarke, K.C. Assessing the effects of land use spatial structure on urban heat islands using HJ-1B remote sensing imagery in Wuhan, China. Int. J. Appl. Earth Obs. 2014, 32, 67-78. [CrossRef]

28. Zhou, W.; Huang, G.; Cadenasso, M.L. Does spatial configuration matter? Understanding the effects of land cover pattern on land surface temperature in urban landscapes. Lands. Urban Plan. 2011, 102, 54-63.

29. Bowler, D.E.; Buyungali, L.; Knight, T.M.; Pullin, A.S. Urban greening to cool towns and cities: A systematic review of the empirical evidence. Landsc. Urban Plan. 2010, 97, 147-155. [CrossRef]

30. Harlan, S.L.; Brazel, A.J.; Prashad, L.; Stefanov, W.L.; Larsen, L. Neighborhood microclimates and vulnerability to heat stress. Soc. Sci. Med. 2006, 63, 2847-2863. [CrossRef] [PubMed]

31. Taha, H. Urban climates and heat islands: Albedo, evapotranspiration, and anthropogenic heat. Energy Build. 1997, 25, 99-103. [CrossRef]

32. Rhinane, H.; Hilali, A.; Berrada, A.; Hakdaoui, M. Detecting Slums from SPOT Data in Casablanca Morocco Using an Object Based Approach. J. Geogr. Inf. Syst. 2011, 3, 217-224. [CrossRef]

33. Owen, K.K.; Wong, D.W. An approach to differentiate informal settlements using spectral, texture, geomorphology and road accessibility metrics. Appl. Geogr. 2013, 38, 107-118. [CrossRef]

34. Guangzhou Economic and Social Development Statistics Bulletin. 2017. Available online: http://www. gdstats.gov.cn/tjzl/tigb / (accessed on 2 March 2018).

35. He, J.B. The Research on the Transformation of Urban Village in Pearl River Delta Developed Area Base on Case Study on the Xihu Village. Master's Thesis, Zhongkai University of Agriculture and Engineering, Guangzhou, China, 2013.

36. Qin, Z.; Dall' Olmo, G.; Karnieli, A.; Berliner, P. Derivation of split window algorithm and its sensitivity analysis for retrieving land surface temperature from NOAA-AVHRR data. J. Geophys. Res. 2001, 106, 22655-22670. [CrossRef]

37. Zhang, Z. Analysis on Heat Island Effect in Coastal Urbanization Area Using Remote Sensing Data: A Case Study in the Qingdao Area. Master's Thesis, Ocean University of China, Qingdao, China, 2013.

38. Xu, H.Q.; Chen, B. An image processing technique for the study of urban heat island changes using different seasonal remote sensing data. Remote Sens. Technol. Appl. 2003, 3, 129-133.

39. Jiménez-Muñoz, J.C.; Sobrino, J.A. A generalized single-channel method for retrieving land surface temperature from remote sensing data. J. Geophys. Res. 2003, 108. [CrossRef]

40. Qin, Z.; Zhang, M.H.; Karnieli, A.; Berliner, P. Mono-window algorithm for retrieving land surface temperature from Landsat TM6 data. Acta Geogr. Sin. 2001, 56, 456-466. 
41. Rozenstein, O.; Qin, Z.; Derimian, Y.; Karnieli, A. Derivation of land surface temperature for Landsat-8 TIRS using a split window algorithm. Sensors 2014, 14, 5768-5780. [CrossRef] [PubMed]

42. Kuffer, M.; Pfeffer, K.; Sliuzas, R. Slums from space-15 years of slum mapping using remote sensing. Remote Sens. 2016, 8, 455. [CrossRef]

43. Haralick, R.M.; Shanmugam, K. Textural features for image classification. IEEE Trans. Syst. Man Cybern. 1973, 6, 610-621. [CrossRef]

44. Zhang, X.Y. RS and GIS-Based Research on Shanghai Land Use/Cover Change with Respect to Thermal Environmental Effect. Master's Thesis, East China Normal University, Shanghai, China, 2006.

45. Huang, X.; Zhang, L. An adaptive mean-shift analysis approach for object extraction and classification from urban hyperspectral imagery. IEEE Trans. Geosci. Remote Sens. 2008, 46, 4173-4185. [CrossRef]

46. Myint, S.W.; Gober, P.; Brazel, A.; Grossman-Clarke, S.; Weng, Q. Per-pixel vs. object-based classification of urban land cover extraction using high spatial resolution imagery. Remote Sens. Environ. 2011, 115, 1145-1161. [CrossRef]

47. Pinho, C.M.; Fonseca, L.M.; Korting, T.S.; Cláudia, C.A.; Kux, H.J.H. Land-cover classification of an intra-urban environment using high-resolution images and object-based image analysis. Int. J. Remote Sens. 2012, 33, 5973-5995. [CrossRef]

48. Baatz, M.; Schäpe, A. Multiresolution segmentation-An optimization approach for high quality multi-scale image segmentation. In Angewandte Geographische Informations-Verarbeitung, XII; Wichmann Verlag: Karlsruhe, Germany, 2000; pp. 12-23.

49. Martinuzzi, S.; Gould, W.A.; González, O.M. Land development, land use, and urban sprawl in Puerto Rico integrating remote sensing and population census data. Lands. Urban Plan. 2007, 79, 288-297. [CrossRef]

50. Razzaghmanesh, M.; Beecham, S.; Salemi, T. The role of green roofs in mitigating Urban Heat Island effects in the metropolitan area of Adelaide, South Australia. Urban For. Urban Green. 2016, 15, 89-102. [CrossRef]

51. Sharma, A.; Conry, P.; Fernando, H.J.S.; Hamlet, A.F.; Hellmann, J.J.; Chen, F. Green and cool roofs to combat urban heating in Chicago: Evaluation with WRF regional climate model. Environ. Res. Lett. 2016, 11, 064004. [CrossRef]

52. Carson, T.B.; Marasco, D.E.; Culligan, P.J.; McGillis, W.R. Hydrological performance of extensive green roofs in New York City: Observations and multi-year modeling of three full-scale systems. Environ. Res. Lett. 2013, 8, 024036. [CrossRef]

53. Berardi, U.; GhaffarianHoseini, A.; GhaffarianHoseini, A. State-of-the-art analysis of the environmental benefits of green roofs. Appl. Energy 2014, 115, 411-428. [CrossRef]

54. Fu, M.X. Research on Urban Village Transformation Basing on Eco-City Building. Master's Thesis, Ocean University of China, Qingdao, China, 2009.

55. Zhao, B. Relevant Health Problems Research on Village-in-City Residents and Environment in Kunming. Master's Thesis, Kun Ming Medical University, Kunming, China, 2011.

56. Jiang, H.Q.; Xie, J.Y.; Biao, L.I.; Xiao, N.; Gu, W.M. Epidemiological characteristics of communicable diseases in an urban village of Shenzhen from 2009 to 2013. J. Trop. Med. 2014, 14, 946-949.

(C) 2018 by the authors. Licensee MDPI, Basel, Switzerland. This article is an open access article distributed under the terms and conditions of the Creative Commons Attribution (CC BY) license (http:/ / creativecommons.org/licenses/by/4.0/). 